\title{
Combined silver Perls's stain for differential staining of ringed sideroblasts and marrow iron
}

\author{
K T Tham, J B Cousar
}

\begin{abstract}
During a study of nucleolar organiser regions, a modified silver stain was found to be a sensitive marker for the iron in ringed sideroblasts, more so than Perls's stain when the marrow iron stores were low. To enhance the usefulness of the silver stain, a combined silver Perls method was developed. This stains the ringed sideroblast iron black and haemosiderin blue, thus rendering the detection of ringed sideroblasts easier even when marrow iron stores are excessive. At the same time, it allows marrow iron content to be evaluated. The silver reagent in this combined method probably shows phosphate rather than the iron present in the abnormal mitochondria in ringed sideroblasts. This facilitates the differential staining of ringed sideroblast "iron" and haemosiderin.
\end{abstract}

(F Clin Pathol 1993;46:766-768)

Evaluation of iron stores in macrophages and abnormal iron deposits in sideroblasts and plasma cells is an important part of bone marrow examination. Perls's Prussian blue stain and its various modifications are traditionally used for this purpose. All iron deposits are stained blue.

During a study of the nucleolar organiser region (NORs) with a silver stain, iron particles in ringed sideroblasts stained black (personal observation). Application of the silver stain to a series of marrow particle sections showed that the stain was more sensitive than Perls's stain for ringed sideroblasts, especially when iron stores were low or absent. ${ }^{1}$ To enhance further the utility of the stain, a combined silver and Perls's (CSP) stain was developed to produce differential staining of ringed sideroblast iron and other iron deposits. The CSP stain was compared with Mallory's modification of Perls's stain for sensitivity. Apart from its diagnostic usefulness, the differential staining capacity of CSP stain may have pathophysiological relevance because it suggests that the chemical composition of iron deposits in mitochondria are different from those in macrophages.

\section{Methods}

Fifty consecutive marrow aspirates were selected without prior knowledge of the diagnoses. Five marrow aspirates known to con- tain ringed sideroblasts were also included in the study. Three were from patients with myelodysplastic syndrome, and two from patients with erythroleukaemia and acute lymphocytic leukaemia, respectively. Paraffin wax sections were stained with a Mallory modification of Perls's stain and also with the CSP silver and Perls's stain and examined microscopically for ringed sideroblasts.

PROCEDURE FOR THE COMBINED SILVER AND PERLS'S STAIN

1 Dewax paraffin sections and bring down to water with decreasing concentrations of ethanol.

2 If the marrow tissue is fixed in fixative containing mercury, remove mercury with Lugol's iodine for 5 minutes. Wash sections with tap water for 3 minutes and rinse with distilled water. Do not use sodium thiosulphate solution to remove iodine.

3 Cover each section with 3 drops (about $0.15 \mathrm{ml}$ ) of a freshly prepared silver staining solution ( 1 volume $50 \%$ silver nitrate, 2 volumes $1 \%$ formic acid in $2 \%$ aqueous gelatin solution).

4 Drop a $40 \times 24 \mathrm{~mm}$ coverslip on the staining solution to spread it evenly.

5 Allow to stain for $\mathbf{4 0}$ minutes in complete darkness.

6 Remove coverslips and rinse sections in distilled water.

7 Stain section with a 1 in 1 mixture of $5 \%$ potassium ferrocyanide and $5 \%$ hydrochloric acid in a Coplin jar for 4 minutes.

8 Wash thoroughly in distilled water.

9 Counterstain with nuclear fast red (Kernechtrot solution) for 3 minutes.

10 Wash in tap water, dehydrate with increasing concentrations of ethanol, clear and mount sections.

\section{Results}

The CSP stain showed the iron in macrophages and plasma cells as coarse blue granules; that in ringed sideroblasts was seen as uniform, small jet-black granules, scattered around the cell nuclei (figure). Sometimes the black granules were clustered focally. Occasionally aggregates of black granules were present in red cells and intercellular spaces adjacent to ringed sideroblasts (fig 1A). Cell nucleoli and lipofuscin were stained dark brown. The stain also worked on marrow smears but not on decalcified marrow aspirates. On marrow smears, the staining tends to be uneven due to variable thickness 
Figure 1 (A) Marrow with increased iron store. Iron in macrophages is stained blue and that in ringed sideroblasts black. Black granules are also present in a few red cells and intercellular spaces (CSP stain).

(B) Fine black granules in early forms of erythroblasts (CSP stain).

(C) Black granules in three ringed sideroblasts and in the cytoplasm of a mitotic cell probably of erythroid lineage (CSP stain).
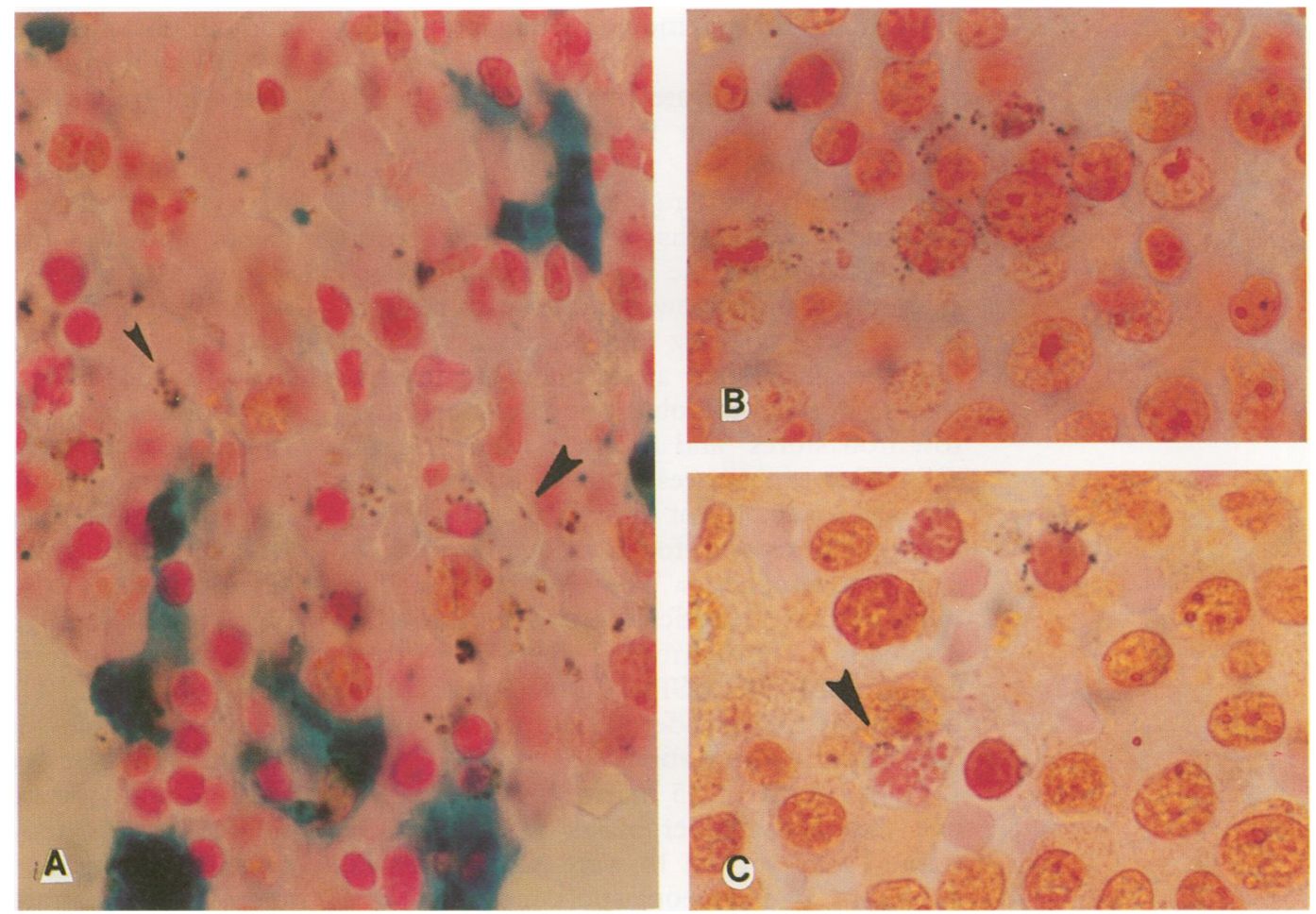

of the smears. For the same reason, quantitation of iron stores and ringed sideroblasts may not be as reliable as when paraffin wax sections of more uniform thickness are used. On the other hand, if marrow smears are well spread and thin, more cytological details and finer iron deposits can be discerned.

All five marrows known to be positive for ringed sideroblasts had increased iron stores. The recognition of ringed sideroblasts was facilitated with the CSP stain because of the differential staining of ringed sideroblast iron and other iron in macrophages or plasma cells (fig 1A). Of the five marrows, four gave equivalent results by both staining methods. In one, more ringed sideroblasts were identified with the CSP stain than with Perls's stain, due to the staining of fine iron granules in the early forms of erythroblasts by the CSP stain in addition to the more mature forms (fig 1B). Cells in mitosis were identifiable as ringed sideroblasts because they contained black granules in the cytoplasm (fig 1C). Perls's stain showed only the more mature ringed sideroblasts with coarser iron particles.

Marrows positive for ringed sideroblasts out of 50 consecutive unknown marrows

\begin{tabular}{|c|c|c|c|c|}
\hline Marrows & $\begin{array}{l}\text { Positivity by } \\
\text { Perls's stain }\end{array}$ & $\begin{array}{l}\text { Positivity by } \\
\text { CSP stain }\end{array}$ & $\begin{array}{l}\text { Iron } \\
\text { stores }\end{array}$ & Diagnoses \\
\hline $\begin{array}{l}1 \\
2\end{array}$ & $\begin{array}{l}+ \\
+\end{array}$ & + & $\begin{array}{l}\text { Increased } \\
\text { Increased }\end{array}$ & $\begin{array}{l}\text { Malignant lymphoma } \\
\text { Small cell carcinoma, lung, S/P } \\
\text { Chemotherapy }\end{array}$ \\
\hline $\begin{array}{l}3 \\
4 \\
5 \\
6 \\
7\end{array}$ & $\begin{array}{l}\overline{-} \\
\overline{-} \\
\overline{-}\end{array}$ & $\begin{array}{l}+ \\
++ \\
+ \\
+ \\
+\end{array}$ & $\begin{array}{l}\text { Increased } \\
\text { Normal } \\
\text { Normal } \\
\text { Normal } \\
\text { Decreased }\end{array}$ & $\begin{array}{l}\text { Plasma cell dyscrasia } \\
\text { Mastocytosis } \\
\text { Focal hypocellularity, cause uncertain } \\
\text { Small cell carcinoma, lung } \\
\text { Acute lymphocytic leukaemia, }\end{array}$ \\
\hline $\begin{array}{l}8 \\
9\end{array}$ & - & + & $\begin{array}{l}\text { Decreased } \\
\text { Decreased }\end{array}$ & $\begin{array}{l}\text { Microcytic anaemia } \\
\text { Acute myelomonocytic leukaemia }\end{array}$ \\
\hline
\end{tabular}

$+=$ occasional ringed sideroblasts present; $++=$ many ringed sideroblasts present; $+++=$ numerous ringed sideroblasts present in many microscopic fields.
Of the 50 consecutive marrow aspirates selected for the study, two marrows had rare ringed sideroblasts demonstrable by both Perls's and CSP stains. Seven marrows had ringed sideroblasts demonstrable only by the CSP stain. The positive results together with iron store content and diagnoses are listed in the table. It was remarkable that in cases 3 and 9, several ringed sideroblasts were seen with the CSP stain but none with Perls's stain. In case 9, electron microscopy also failed to identify ringed sideroblasts.

\section{Discussion}

The silver stain used is a modification of that first introduced by Howell and Black ${ }^{1}$ and improved by Ploton and colleagues ${ }^{2}$ to demonstrate NORs. It stains iron, calcium, melanin, lipofuscin and fungal elements (personal observation), in addition to the argyrophilic protein associated with NORs. Therefore, it is not a specific stain. Nevertheless, in marrow tissues the pattern of perinuclear black granules in erythroblasts is so characteristic that misidentification is unlikely.

The use of coverslips in the staining procedures minimises the volume of silver staining solution, ensures even staining, and reduces non-specific silver precipitate. The potassium ferrocyanide/hydrochloric acid solution used in step 7 of the staining procedure serves both as a differentiating agent for the silver stain and as a staining agent for iron in macrophages and plasma cells. If overdifferentiated, some or all of the granules in ringed sideroblasts turned blue. As a further modification of the previous method ${ }^{3}$ the sodium thiosulphate solution, which reduces the sensitivity of the silver stain, may be omitted 
from the mercury-removing sequence without adverse effect.

Our results indicate that the CSP stain is more sensitive than Perls's stain alone for ringed sideroblasts, particularly when the marrow iron stores are low or absent. A previous study reported that a simple silver stain is also sufficient for the identification of ringed sideroblasts in marrow aspirates with reduced iron stores. ${ }^{3}$ Nikicicz and Norback reported that the silver stain alone could be used to assess not only sideroblast but also bone marrow iron stores ${ }^{4}$ and to study other marrow cells. ${ }^{5}$ Our experiences indicate, however, that the silver stain is not specific for iron and is not recommended for the identification of iron in macrophages. The CSP stain permits more specific identification and evaluation of iron stores. Furthermore, when iron stores are excessive, the CSP stain facilitates identification of ringed sideroblasts because of its differential staining ability.

In our study of 55 marrows 14 were positive for ringed sideroblasts with the CSP stain. Twelve of the 14 were associated with neoplastic or pre-neoplastic conditions. One of the two exceptions was from a 68 year old patient with a focally hypocellular marrow of uncertain importance. The other was from a 82 year old patient with iron deficiency anaemia receiving multiple medications for hypertension and chronic obstructive pulmonary disease.

Iron in ringed sideroblasts is located in the mitochondria. Using energy dispersive $x$ ray analysis of the mitochondria, Grasso and colleagues detected a consistent presence of iron and phosphorus. ${ }^{6}$ They suggested that iron was present in the form of ferric phosphate. Other elements variably detected included calcium, lead, potassium and zinc. No phosphorus was detected in normal or unaffected mitochondria in the same section. Ferritin and haemosiderin contained relatively much less phosphorus. Their findings suggest a possible staining mechanism for the silver stain; the stain shows the phosphate and not the iron moiety of ferric phosphate. A similar mechanism is seen with von Kossa's stain which demonstrates the phosphate and carbonate but not the calcium in bone. ${ }^{7}$ Both stains do not work on decalcified material. Von Kossa's stain, however, does not demonstrate ringed sideroblasts (personal observation). The differential staining capability of the CSP stain supports the above hypothesis. Haemosiderin in macrophages is stained blue because of the excess of iron over phosphate. In ringed sideroblasts the black staining of the phosphate moiety masks the blue staining of the iron moiety. If overdifferentiated, the black stain is removed and the blue stain becomes visible. In iron deficiency the abnormal mitochondria in ringed sideroblasts may contain phosphates of other elements in addition to or instead of iron. Therefore, Perls's stain will be weakly positive or negative but silver stain will still be distinctively positive. This explains the greater sensitivity of the silver stain for ringed sideroblasts in iron deficiency and also explains the loss of ringed sideroblasts in severe iron deficiency reported by Stavem and colleagues. ${ }^{8}$ If the silver stain is specific for abnormal mitochondrial "iron" deposits, then even a few black granules in an erythroblast may be enough to identify it as a ringed sideroblast, as opposed to the requirement of finding at least five blue granules encircling more than one third of the circumference of the nucleus to identify a ringed sideroblast by Perls's stain. ${ }^{9}$ The presence of black cytoplasmic granules may help to identify a "blast" or mitotic figure as an erythroid cell (figs 1B and C). Furthermore, the presence of multiple black granules in red cells and intercullular spaces (fig 1A) may be an indication to search carefully for ringed sideroblasts as they may be derived from ringed sideroblasts. The ability of the silver stain to demonstrate fungal elements adds to its usefulness.

\section{Conclusion}

The CSP stain is more sensitive than Perls's stain for ringed sideroblasts. It also permits evaluation of iron stores. The silver reagent of the CSP stain probably demonstrates phosphate and not iron in mitochondria of ringed sideroblasts. If it is a specific marker for ringed sideroblasts, then the presence of a few black granules may be enough to identify an erythroblast as a ringed sideroblast, and the presence of black granules in red cells and the intercellular spaces may be an indication to search carefully for ringed sideroblasts.

1 Howell WM, Black DA: Controlled silver staining of nucleolus organiser regions with a protective colloidal developer: a one-step method. Experientia 1980;36: 1014-5.

2 Ploton D, Menager M, Jeannesson P, Himber G, Pigeon $F$, Adnet JJ. Improvement in the staining and in the visualization of the argyrophilic proteins of the nucleolar organizer region at the optical level. Histochem $\mathcal{f}$ 1986;18:5-14.

3 Tham KT, Cousar JB, Macon WR. Silver stain for ringed sideroblasts: Am f Clin Pathol 1990;94:73-6.

4 Nikicicz EP, Norback DH. The use of a collodial silver (AgNOR) method in assessing bone marrow iron stores and sideroblasts. Mod Pathol 1991;4:363-7.

5 Nikicicz EP, Norback DH. Argyrophilic nucleolar organiser region (AgNOR) staining in bone marrow cells: iser region (AgNOR) staining

6 Grasso JA, Myers TJ, Hines JD, Sullivan AL. Energy-dispersive X-ray analysis of the mitochondria of sideropersive X-ray analysis of the mitochondria of

7 Bancroft JD. Histochemical techniques. 2nd edn. London Butterworths, 1975:211.

8 Stavem P, Rorvik TO, Rootwelt K, Josefsen JO. Severe iron deficiency causing loss of ring sideroblasts. Scand $\mathcal{Y}$ Haematol 1983;31:389-91.

9 Juneja SK, Imbert $M$, Sigaux F, Jouault H, Sultan C: Prevalence and distribution of ringed sideroblasts in primary myelodysplastic syndromes. $\mathcal{F}$ Clin Pathol 1983;36 $566-9$. 